\title{
Non-conformal holographic Gauss-Bonnet hydrodynamics
}

\author{
Alex Buchel \\ Department of Applied Mathematics, Department of Physics and Astronomy, \\ University of Western Ontario, London, Ontario N6A 5B7, Canada \\ Perimeter Institute for Theoretical Physics, \\ Waterloo, Ontario N2J 2W9, Canada \\ E-mail: abuchel@perimeterinstitute.ca
}

ABSTRACT: We study hydrodynamics of four-dimensional non-conformal holographic plasma with non-equal central charges $c \neq a$ at the ultraviolet fixed point. We compute equation of state, the speed of sound waves, transport coefficients (shear and bulk viscosities), and discuss causality. We study the asymptotic character of the hydrodynamic series for the homogeneous and isotropic expansion of the plasma. We perform computations for finite $c-a \neq 0$, but to leading nonvanishing order in the conformal symmetry breaking coupling.

KeYwords: AdS-CFT Correspondence, Gauge-gravity correspondence, Holography and quark-gluon plasmas

ARXIV EPRINT: 1801.06165 


\section{Contents}

1 Introduction 1

2 Non-conformal Gauss-Bonnet holographic model 2

3 Hydrodynamic transport: shear and bulk viscosities 5

4 Homogeneous and isotropic expansion of the plasma $\quad 8$

$\begin{array}{lll}5 & \text { Causality } & 13\end{array}$

6 Conclusion $\quad 15$

\section{Introduction}

Holographic correspondence $[1,2]$ provided an opportunity to explore near- and far-fromequilibrium properties of strongly coupled gauge theories [3,4] . Recently, ${ }^{1}$ there was been a revival of interest in holographic models of conformal hydrodynamics, where the dual gauge theory has a finite 't Hooft coupling, or the non-equal central charges $c-a \neq 0[12-$ 16]. We should emphasize that in string-theoretic top-down holographic constructions only the leading finite 't Hooft coupling corrections are known, and only for $\mathcal{N}=4$ supersymmetric Yang-Mills [17-19]. Since on the gravitational side of the duality these corrections correspond to higher-derivative corrections in the equations of motion, they can not, even in principle, be treated beyond infinitesimally small approximation. On the contrary, corrections due to non-equal central charges of a four-dimensional conformal gauge theory are encoded in the gravitational Gauss-Bonnet (GB) coupling constant $\lambda_{\mathrm{GB}}$ (see section 2 for details), which maintains the second-order character of the equations of motion, thus allowing for the holographic analysis to be extended to finite values of the coupling. Although at a technical level holographic models can be explored for finite $\lambda_{\mathrm{GB}}$, alas, fundamentally, these models are still consistent at best for infinitesimal values of the GB coupling [22].

The purpose of this paper is to report the results of the study of hydrodynamics of holographic non-conformal models with a Lagrangian density $\mathcal{L}$. We consider holographic renormalization group ( $\mathrm{RG}$ ) flows close to the ultraviolet (UV) fixed point, with Lagrangian density $\mathcal{L}_{\text {CFT }}$ perturbed by a relevant operator of $\mathcal{O}_{\Delta}$ of dimension $\Delta$ :

$$
\mathcal{L}=\mathcal{L}_{\mathrm{CFT}}+\lambda_{4-\Delta} \mathcal{O}_{\Delta}
$$

\footnotetext{
${ }^{1}$ See $[5-11]$ for the early work.
} 
We allow for a finite difference of the UV CFT central charges: $c-a \neq 0$. By 'close' we mean that the local temperature $T$ of the dual gauge theory plasma is much higher than the conformal symmetry breaking scale, i.e.,

$$
\frac{\left|\lambda_{4-\Delta}\right|}{T^{4-\Delta}} \ll 1
$$

The paper is organized as follows. We introduce the model in section 2 . We discuss holographic renormalization for the RG flows with $\Delta=\{2,3\}$, and present the equilibrium equations of state of the dual plasma. In section 3 we first compute the shear viscosity from the retarded two-point correlation function of the equilibrium stress-energy tensor of the boundary plasma. Next, we compute the dispersion relation of the sound waves in plasma, and extract the speed of sound and the bulk viscosity. In section 4 we study the asymptotic character of the entropy production for the homogeneous and the isotropic expansion of the plasma to all orders in the gradient expansion parameter. In section 5 we discuss microscopic causality of the model. We conclude in section 6 .

The computational frameworks are well documented in the literature and will not be reviewed here. For reader's convenience we collect below references to the papers adopted in the analysis:

- holographic renormalization - [23];

- shear viscosity from the retarded stress-energy tensor correlation functions - [24];

- sound waves in holographic plasma - $[25,26]$;

- beyond hydrodynamics for homogeneous and isotropic expansions - [27];

- microscopic causality — [28].

\section{Non-conformal Gauss-Bonnet holographic model}

We define the boundary gauge theory through its dual Gauss-Bonnet gravitational bulk model:

$$
\begin{aligned}
\mathcal{I} & =\frac{1}{2 \ell_{P}^{3}} \int_{\mathcal{M}_{5}} d^{5} x \sqrt{-g}\left[\mathcal{L}_{\mathrm{CFT}}+\delta \mathcal{L}\right], \\
\mathcal{L}_{\mathrm{CFT}} & =\frac{12}{L^{2}}+R+\frac{\lambda_{\mathrm{GB}}}{2} L^{2}\left(R^{2}-4 R_{\mu \nu} R^{\mu \nu}+R_{\mu \nu \rho \sigma} R^{\mu \nu \rho \sigma}\right), \\
\delta \mathcal{L} & =-\frac{1}{2}(\partial \phi)^{2}-\frac{1}{2} m^{2} \phi^{2},
\end{aligned}
$$

where $\mathcal{L}_{\mathrm{CFT}}$ is the bulk Lagrangian of the UV conformal fixed point with central charges [20, 29]

$$
\begin{aligned}
& c=\frac{\pi^{2}}{2^{3 / 2}} \frac{L^{3}}{\ell_{P}^{3}}\left(1+\sqrt{1-4 \lambda_{\mathrm{GB}}}\right)^{3 / 2} \sqrt{1-4 \lambda_{\mathrm{GB}}}, \\
& a=\frac{\pi^{2}}{2^{3 / 2}} \frac{L^{3}}{\ell_{P}^{3}}\left(1+\sqrt{1-4 \lambda_{\mathrm{GB}}}\right)^{3 / 2}\left(3 \sqrt{1-4 \lambda_{\mathrm{GB}}}-2\right),
\end{aligned}
$$


and $\delta \mathcal{L}$ is the conformal symmetry breaking perturbation realizing (1.1) for

$$
m^{2} L^{2} \beta_{2}=\Delta(\Delta-4), \quad \beta_{2} \equiv \frac{1}{2}+\frac{1}{2} \sqrt{1-4 \lambda_{\mathrm{GB}}}, \quad \lambda_{\mathrm{GB}}=\beta_{2}-\beta_{2}^{2} .
$$

The UV conformal fixed point is a causal gauge theory, provided [28, 30],

$$
-\frac{7}{36} \leq \lambda_{\mathrm{GB}} \leq \frac{9}{100} \quad \Longleftrightarrow \quad-\frac{1}{2} \leq \frac{c-a}{c} \leq \frac{1}{2} .
$$

In what follows, without the loss of generality, we set $L=1$.

To study equilibrium thermal states of the model we use the bulk metric ansatz

$$
d s_{5}^{2}=\frac{r_{h}^{2}}{x}\left(-f_{1} \beta_{2} d t^{2}+\sum_{i=1}^{3} d x_{i}^{2}\right)+\frac{1}{f_{2}} \frac{d x^{2}}{4 x^{2}},
$$

where the metric warp factors $f_{i}$ and the bulk scalar $\phi$ are functions of the radial coordinate $x$ only,

$$
x \in(0,1) .
$$

The asymptotically AdS boundary is located at $x=0$ and the regular Schwarzschild horizon at $x=1$. Parameter $r_{h}$ is related to the Hawking temperature of the horizon. Asymptotically near the boundary,

$$
\phi=\delta_{\Delta} \times \begin{cases}x^{1 / 2}+\mathcal{O}\left(x^{3 / 2}\right), & \Delta=3, \\ x \ln x+\mathcal{O}(x), & \Delta=2,\end{cases}
$$

with the non-normalizable component of the scalar field $\delta_{\Delta}$ identified with the corresponding coupling constant $\lambda_{4-\Delta}$ as

$$
\lambda_{4-\Delta}=\delta_{\Delta} r_{h}^{4-\Delta}
$$

For a vanishing source $\delta_{\Delta}=0$, the above gravitational background, explicitly,

$$
f_{1}=f_{2} \equiv f(x)=\frac{1-\sqrt{1-4\left(\beta_{2}-\beta_{2}^{2}\right)\left(1-x^{2}\right)}}{2\left(\beta_{2}-\beta_{2}^{2}\right)}, \quad \phi=0,
$$

describes the gravitational dual to a thermal state of the UV conformal fixed point. For $\delta_{\Delta} \neq 0$ the background geometry can be easily constructed numerically.

The equilibrium thermal state of the boundary gauge theory is characterized by the temperature $T$, the entropy density $s$, the pressure $P$ and the energy density $\mathcal{E}$. The entropy density is the Bekenstein or the Wald entropy ${ }^{2}$ of the background geometry:

$$
s=\frac{2 \pi r_{h}^{3}}{\ell_{P}^{3}},
$$

the temperature is related to the surface gravity $\kappa$ at the horizon,

$$
T=\frac{\kappa}{2 \pi}=\left.\frac{r_{h} \beta_{2}^{1 / 2}}{\pi} \frac{\sqrt{f_{1}^{\prime} f_{2}^{\prime}}}{2}\right|_{x=1} .
$$

\footnotetext{
${ }^{2}$ Both are the same for the GB gravity, see [31].
} 
To compute the energy density and the pressure, one needs to holographically renormalize the model. This step involves specifying the generalized Gibbons-Hawking term at the regularization boundary $\partial \mathcal{M}_{5}, S_{\mathrm{GH}}$ (see e.g., [14]),

$$
S_{\mathrm{GH}}=-\frac{1}{\ell_{P}^{3}} \int_{\partial \mathcal{M}_{5}} d^{4} x \sqrt{-\gamma}\left[K+\left(\beta_{2}-\beta_{2}^{2}\right)\left(J-2 G_{\gamma}^{\mu \nu} K_{\mu \nu}\right)\right] .
$$

Here $\gamma_{\mu \nu}=g_{\mu \nu}-n_{\mu} n_{\nu}$ is the induced metric on the boundary, $n^{\mu}$ is the unit outwards vector to the boundary and $G_{\gamma}^{\mu \nu}$ is the induced Einsteins tensor on the boundary. The extrinsic curvature tensor is

$$
K_{\mu \nu}=-\frac{1}{2}\left(\nabla_{\mu} n_{\nu}+\nabla_{\nu} n_{\mu}\right)
$$

$K$ is its trace and the tensor $J_{\mu \nu}$ is defined as

$$
J_{\mu \nu}=\frac{1}{3}\left(2 K K_{\mu \rho} K_{\nu}^{\rho}\right)+K_{\rho \sigma} K^{\rho \sigma} K_{\mu \nu}-2 K_{\mu \rho} K^{\rho \sigma} K_{\sigma \nu}-K^{2} K_{\mu \nu},
$$

with $J$ being the trace of the latter. Additionally, we must include the counter-term action at the regularization boundary (located at the radial position $x=\epsilon$ ):

$$
\begin{aligned}
S_{\text {c.t. }} & =\frac{1}{\ell_{P}^{3}} \int_{\partial \mathcal{M}_{5}} d^{4} x \sqrt{-\gamma}\left[\mathcal{L}_{\text {c.t.,CFT }}+\mathcal{L}_{\text {c.t. }, \Delta}\right], \\
\mathcal{L}_{\text {c.t.,CFT }} & =-\left(2 b_{2}^{1 / 2}+b_{2}^{-1 / 2}\right)+\left(\frac{1}{2} b_{2}^{3 / 2}-\frac{3}{4} \beta_{2}^{1 / 2}\right) R_{\gamma}+\left(\frac{1}{8} \beta_{2}^{5 / 2}-\frac{1}{16} \beta_{2}^{3 / 2}\right) \mathcal{P}_{2, \gamma} \ln \epsilon, \\
\mathcal{L}_{\text {c.t., } \Delta} & = \begin{cases}-\frac{1}{4} \beta_{2}^{-1 / 2} \phi^{2}-\frac{\beta_{2}^{-1 / 2}}{48\left(2 \beta_{2}-1\right)} \phi^{4} \ln \epsilon-\frac{\beta_{2}^{1 / 2}}{48} R_{\gamma} \phi^{2} \ln \epsilon \quad, \quad \Delta=3, \\
-\frac{1}{2} \beta_{2}^{-1 / 2} \phi^{2}-\frac{1}{2} \beta_{2}^{-1 / 2} \phi^{2} \frac{1}{\ln \epsilon} \quad \Delta=2,\end{cases}
\end{aligned}
$$

where we separated the counterterms necessary to renormalize conformal fixed point $\mathcal{L}_{\text {c.t. }, C F T}$, and the deformation-dependent set of counterterms $\mathcal{L}_{\text {c.t., } \Delta}$. Here ${ }^{3} R_{\gamma}^{\mu \nu}$ is the induced Ricci tensor on the regularization boundary and $R_{\gamma}$ is its trace, and

$$
\mathcal{P}_{2, \gamma}=\mathcal{P}_{\gamma}^{\mu \nu} \mathcal{P}_{\mu \nu, \gamma}-\left(\gamma^{\mu \nu} \mathcal{P}_{\mu \nu}\right)^{2}, \quad \mathcal{P}_{\gamma}^{\mu \nu}=R_{\gamma}^{\mu \nu}-\frac{1}{6} R_{\gamma} \gamma^{\mu \nu}
$$

In practice, we compute the thermodynamic characteristics of the equilibrium state to order $\mathcal{O}\left(\delta_{\Delta}^{2}\right)$ inclusive. Thus, we parameterize the non-conformal equation of state as

$$
c_{s}^{2}-\frac{1}{3}=\left(\frac{\lambda_{4-\Delta}}{T^{4-\Delta}}\right)^{2} \mathcal{F}_{\Delta}\left(\lambda_{\mathrm{GB}}\right)
$$

where

$$
c_{s}^{2}=\frac{\partial P}{\partial \mathcal{E}}
$$

is the speed of the sound waves in plasma. The results for $\mathcal{F}_{\Delta}\left(\lambda_{\mathrm{GB}}\right)$ in the GB causal window (2.4) are presented in figure 1 for $\Delta=3$ (left panel) and $\Delta=2$ (right panel).

\footnotetext{
${ }^{3}$ The terms involving the induced Ricci tensor are necessary to compute the retarded correlation functions of the stress-energy tensor even for the Minkowski boundary metric.
} 

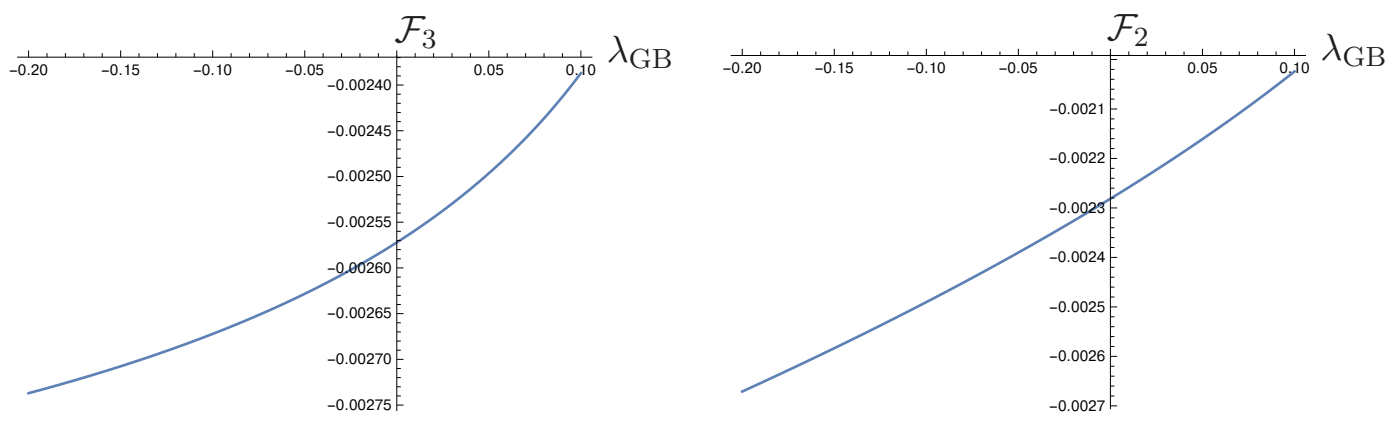

Figure 1. Parameterization of the equilibrium equation of state of the holographic non-conformal GB model (2.1) with the equation (2.17) for deformation of the UV conformal fixed point with $\Delta=3$ (left panel) and $\Delta=2$ (right panel) operators. Note that $\mathcal{F}_{\Delta} \propto+\left(c_{s}^{2}-1 / 3\right)$ and so, within the causality window, the speed of sound is bounded from above by its conformal value.
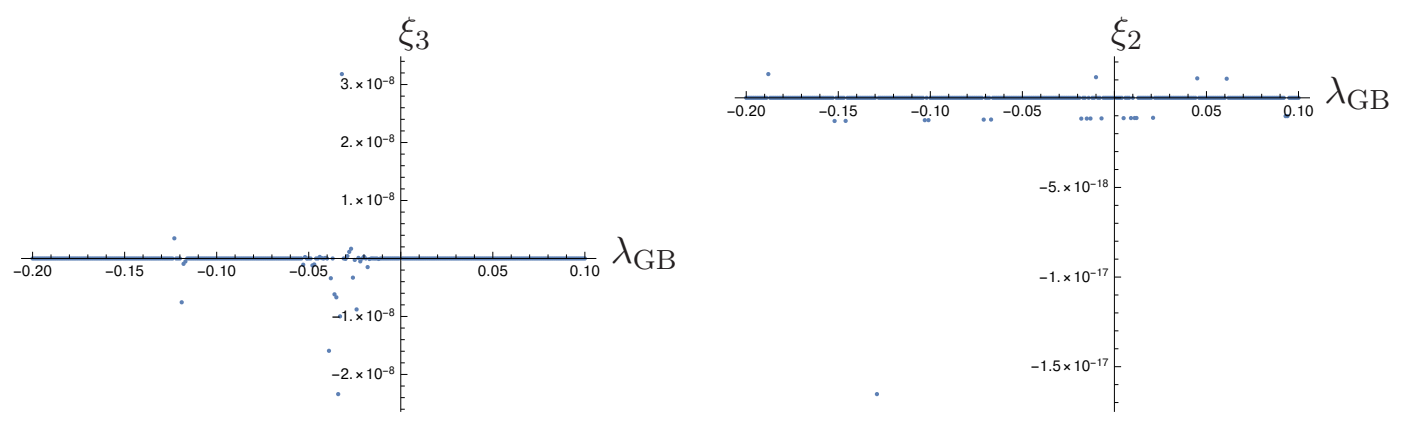

Figure 2. Numerical tests of the first law of the thermodynamics for the non-conformal GB RG flows induced by $\Delta=3$ operator (left panel) and $\Delta=2$ operator (right panel). See (2.19) for the parameterization of the deviation $\xi_{\Delta}$.

While the basic thermodynamic relation, $F$ is the free energy density,

$$
F=-P=\mathcal{E}-s T
$$

is satisfied automatically, the first law of thermodynamics, $d \mathcal{E}=T d s$, does not: it provides an important test on our numerical data. In figure 2 we present tests of the first law of the thermodynamics

$$
\xi_{\Delta}\left(\lambda_{\mathrm{GB}}\right) \equiv\left(\frac{T^{4-\Delta}}{\lambda_{4-\Delta}}\right)^{2} \times \frac{1}{s}\left(\frac{d \mathcal{E}}{d T}-T \frac{d s}{d T}\right)
$$

for $\Delta=3$ (left panel) and $\Delta=2$ (right panel) within the GB causal window (2.4).

\section{Hydrodynamic transport: shear and bulk viscosities}

The most straightforward way to compute the shear viscosity $\eta$ of the model is to, following [24], compute the on-shell renormalized boundary action

$$
\begin{aligned}
S_{\text {renom }}\left[h_{12}(t)\right] & =\mathcal{I}+S_{\mathrm{GB}}+\left.S_{c . t .}\right|_{d s_{4}^{2}}, \\
d s_{4}^{2} & \equiv \hat{\gamma}_{\alpha \beta} d x^{\alpha} d x^{\beta} \equiv-d t^{2}+\sum_{i=1}^{3} d x_{i}^{2}+2 h_{12}(t) d x_{1} d x_{2},
\end{aligned}
$$



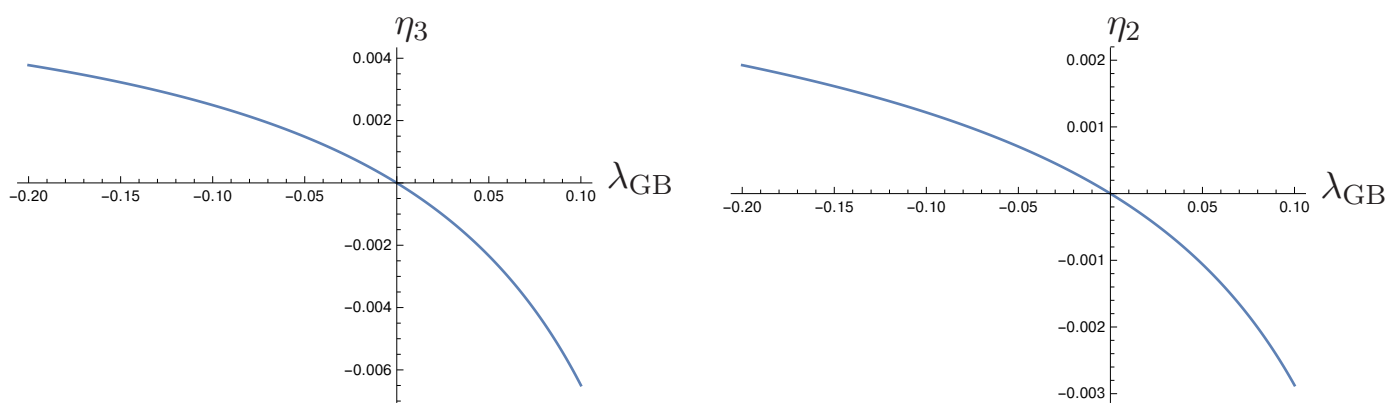

Figure 3. Corrections to the shear viscosity for the non-conformal GB RG flows induced by dimension $\Delta=3$ operator (left panel) and $\Delta=2$ operator (right panel). See (3.5) for the parameterization of the corrections.

(see (2.1), (2.12) and (2.15)) to quadratic order in the boundary metric source term $h_{12}(t)$. The thermal expectation value of the boundary stress-energy tensor

$$
\left.\left\langle T^{\alpha \beta}\right\rangle\right|_{T}=\frac{2}{\sqrt{-\hat{\gamma}}} \frac{\delta S_{\text {renom }}}{\delta \hat{\gamma}_{\alpha \beta}},
$$

in the low frequency limit, i.e.,

$$
T\left|\frac{\dot{h}_{12}}{h_{12}}\right| \ll 1,
$$

then has the off-diagonal component

$$
T^{12}=\left(-P h_{12}-\eta \dot{h}_{12}+\mathcal{O}\left(\ddot{h}_{12}\right)\right)+\mathcal{O}\left(h_{12}^{2}\right),
$$

allowing for the extraction of the shear viscosity. Results of this tedious computation, to order $\mathcal{O}\left(\delta_{\Delta}^{2}\right)$, in the parameterization

$$
\frac{\eta}{s}=\frac{\left(2 \beta_{2}-1\right)^{2}}{4 \pi}\left(1+\eta_{\Delta}\left(\lambda_{\mathrm{GB}}\right)\left(\frac{\lambda_{4-\Delta}}{T^{4-\Delta}}\right)^{2}\right)
$$

are presented in figure 3 for non-conformal RG flow with $\Delta=3$ (left panel) and $\Delta=2$ (right panel). Note that $\lambda_{4-\Delta}=0$ result reproduced computations of [10]; furthermore, $\eta_{\Delta}\left(\lambda_{\mathrm{GB}}=0\right)=0$, reflecting the universality of the shear viscosity [32].

The spectrum of long-wavelength fluctuations in near equilibrium plasma includes longitudinal (sound) waves with the dispersion relation:

$$
\begin{aligned}
& \mathfrak{w}= \pm c_{s} \mathfrak{q}-2 \pi i \frac{\eta}{s}\left(\frac{2}{3}+\frac{\zeta}{2 \eta}\right) \mathfrak{q}^{2}+\mathcal{O}\left(\mathfrak{q}^{3}\right) \\
& \mathfrak{w} \equiv \frac{\omega}{2 \pi T}, \quad \mathfrak{q}=\frac{|\vec{q}|}{2 \pi T}
\end{aligned}
$$

We proceed computing the spectrum of sound waves in model (2.1) following [25]. To this end we parameterize the transport coefficients as

$$
c_{s}^{2}=\frac{1}{3}+\left(\frac{\lambda_{4-\Delta}}{T^{4-\Delta}}\right)^{2} \hat{\mathcal{F}}_{\Delta}\left(\lambda_{\mathrm{GB}}\right), \quad \frac{\zeta}{\eta}=\left(\frac{\lambda_{4-\Delta}}{T^{4-\Delta}}\right)^{2} \zeta_{\Delta}\left(\lambda_{\mathrm{GB}}\right) .
$$



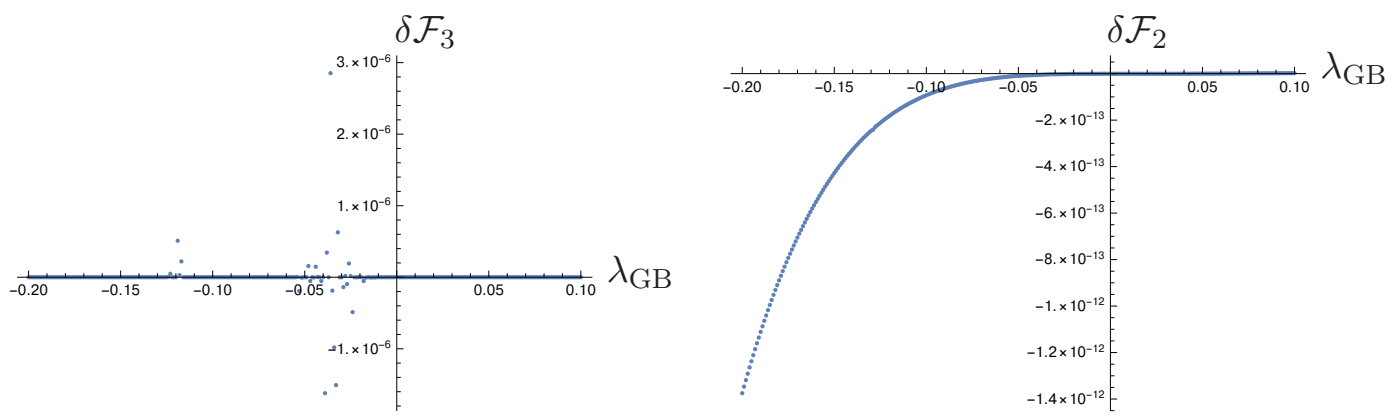

Figure 4. Consistency test on extracting speed of the sound waves from the equation of state (2.18) and directly from the dispersion relation (3.6), see (3.9), for $\Delta=3$ RG flow (left panel) and $\Delta=2$ RG flow (right panel).
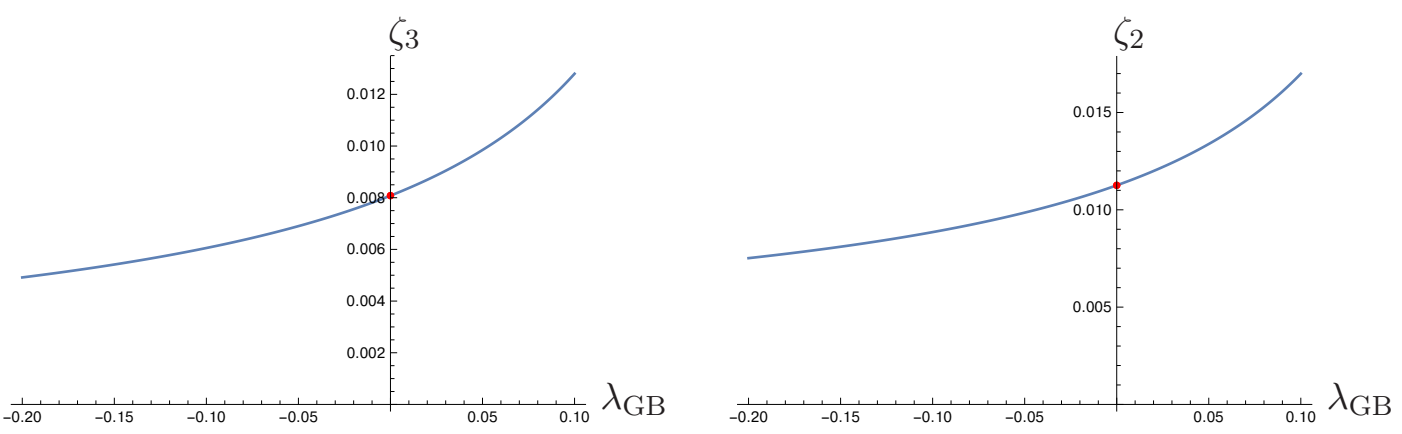

Figure 5. Bulk viscosity of the holographic RG flows induced by dimension $\Delta=3$ (left panel) and $\Delta=2$ (right panel) operators, see (3.7). The red dots indicate the $\lambda_{\mathrm{GB}}=0$ results obtained in $[25,26]$.
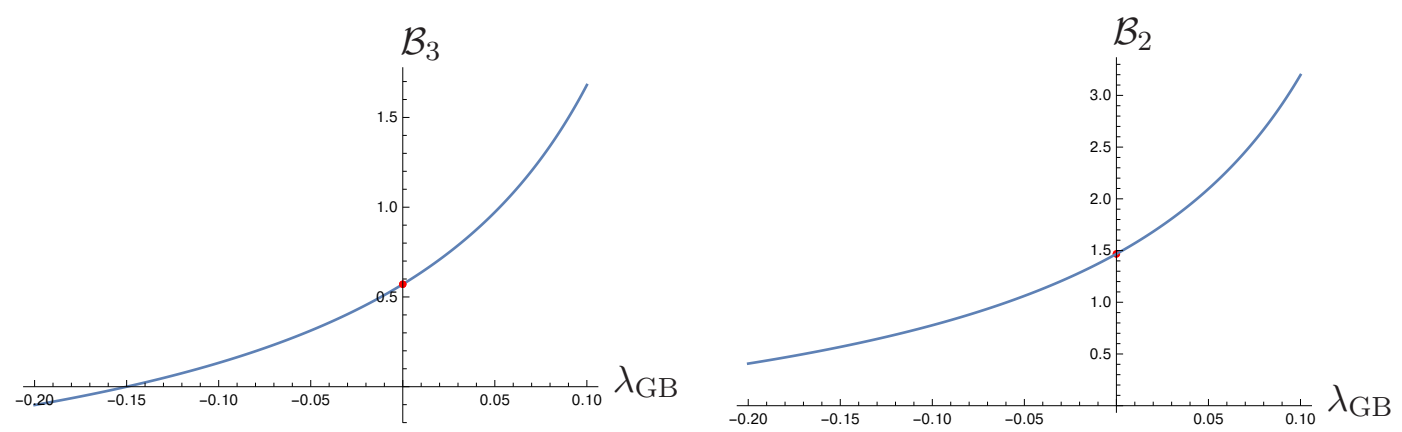

Figure 6. Holographic bulk viscosity bound for RG flows induced by dimension $\Delta=3$ (left panel) and $\Delta=2$ (right panel) operators. The bound is violated whenever $\mathcal{B}_{\Delta}<0$. Red dots represent the bound at $\lambda_{\mathrm{GB}}=0$, see (3.11).

Of course, consistency of the hydrodynamics requires that

$$
\hat{\mathcal{F}}_{\Delta}\left(\lambda_{\mathrm{GB}}\right)=\mathcal{F}_{\Delta}\left(\lambda_{\mathrm{GB}}\right),
$$

where the latter is introduced from the equilibrium equation of state of the plasma, following (2.17) and (2.18). Figure 4 presents

$$
\delta \mathcal{F}_{\Delta}\left(\lambda_{\mathrm{GB}}\right) \equiv \frac{\hat{\mathcal{F}}_{\Delta}}{\mathcal{F}_{\Delta}}-1
$$


- an important consistency check on our numerical results for RG flows with $\Delta=3$ (left panel) nd $\Delta=2$ (right panel).

To extract the bulk viscosity coefficients $\zeta_{\Delta}$, following (3.7), one has to use the results for the shear viscosity, see (3.5). Figure 5 presents $\zeta_{\Delta}$ for RG flow with $\Delta=3$ (left panel) and $\Delta=2$ (right panel). The red dots represent $\lambda_{\mathrm{GB}}=0$ results obtained in [25, 26].

We conclude this section commenting on the holographic bulk viscosity bound [33],

$$
\frac{\frac{\zeta}{\eta}}{2\left(\frac{1}{3}-c_{s}^{2}\right)}-1 \equiv \mathcal{B}_{\Delta}\left(\lambda_{\mathrm{GB}}\right) \geq 0 .
$$

Note that [33]

$$
\left.\mathcal{B}_{\Delta}\right|_{\lambda_{\mathrm{GB}}=0}= \begin{cases}\frac{\pi}{2}-1, & \Delta=3, \\ \frac{\pi^{2}}{4}-1, & \Delta=2 .\end{cases}
$$

Results for $\mathcal{B}_{\Delta}$ are presented in figure 6 for holographic RG flows with $\Delta=3$ (left panel) and $\Delta=2$ (right panel). Note that the bound is violated, within the causal window of the model (2.4) for the RG flow induced by $\Delta=3$ operator (but not in the $\Delta=2$ case). The violation happens in the theories with the UV fixed point with $a-c>0$ central charges. ${ }^{4}$ This is not the first known violation of the bulk viscosity bound: see [34] for the violation of the bound in a top-down model of the gauge/gravity correspondence.

\section{Homogeneous and isotropic expansion of the plasma}

We study in this section homogeneous and isotropic expansion of the non-conformal plasma defined via the dual gravitational action (2.1). We follow discussion in [27]. The purpose of the analysis is twofold:

- we would like to have an independent computation of the bulk viscosity;

- we would like to understand the interplay between the large-order behavior of the hydrodynamic expansion and causality.

Homogeneous and isotropic expansion of the boundary gauge theory plasma can be studied placing the theory in Friedmann-Lemaitre-Robertson-Walker (FLRW) Universe with zero spatial curvature:

$$
d s_{4}^{2}=\hat{\gamma}_{\alpha \beta} d x^{\alpha} d x^{\beta}=-d t^{2}+a(t)^{2} \sum_{i=1}^{3} d x_{i}^{2} .
$$

In the FLRW geometry the matter expansion is locally static $u^{\alpha}=(1,0,0,0)$ though it possesses a nonzero expansion rate $\Theta \equiv \nabla_{\alpha} u^{\alpha}=3 \dot{a} / a$. The corresponding gravitational geometry is best to analyze in infalling Eddington-Finkelstein coordinates:

$$
d s_{5}^{2}=2 d t(d r-A d t)+\Sigma^{2} \sum_{i=1}^{3} d x_{i}^{2} .
$$

\footnotetext{
${ }^{4}$ The shear viscosity bound [35] is violated for CFTs with $c-a>0$ [10].
} 
Here, the bulk scalar field $\phi$ and the metric warp factors $A, \Sigma$ depend only on $\{r, t\}$. The near-boundary $r \rightarrow \infty$ asymptotic behaviour of the metric and the scalar encode the boundary metric scale factor $a(t)$ and the coupling constant $\lambda_{4-\Delta}$, $\sec ^{5}(1.1)$,

$$
\begin{aligned}
& \Sigma=\frac{a}{r}+\mathcal{O}\left(r^{-1}\right), \quad A=\frac{r^{2}}{2 \beta_{2}}-\frac{\dot{a} r}{a}+\mathcal{O}\left(r^{0}\right), \\
& \phi=\lambda_{4-\Delta}\left\{\begin{array}{l}
\frac{1}{r}+\mathcal{O}\left(r^{-2}\right), \\
-\frac{\ln r^{2}}{r^{2}}+\mathcal{O}\left(r^{-2}\right),
\end{array}=3=2 .\right.
\end{aligned}
$$

As in [27] we identify the non-equilibrium entropy density $s$ with the Bekenstein-Hawking entropy of the apparent horizon in the geometry (4.2),

$$
a^{3} s=\left.\frac{2 \pi}{\ell_{P}^{3}} \Sigma^{3}\right|_{r=r_{h}},
$$

where $r_{h}$ is the location of the apparent horizon determined from $\left.d_{+} \Sigma\right|_{r=r_{h}}=0$ with $d_{+} \equiv \partial_{t}+A \partial_{r}$, see [4]. Taking the time derivative of the entropy density and using holographic equations of motion we find

$$
\frac{d\left(a^{3} s\right)}{d t}=\left.\frac{4 \pi}{\ell_{P}^{3}}\left(\Sigma^{3}\right)^{\prime} \frac{\left(d_{+} \phi\right)^{2}}{24-m^{2} \phi^{2}}\right|_{r=r_{h}} .
$$

Following [36] it is easy to show that the gravitational equations of motion guarantee that the entropy production rate is nonnegative. In the hydrodynamics regime, i.e., for small Knudsen numbers $K_{N}=\Theta / T \ll 1$,

$$
\left.\frac{d}{d t} \ln \left(a^{3} s\right)\right|_{\text {hydro }} \approx \frac{1}{T}(\nabla \cdot u)^{2} \frac{\zeta}{s}
$$

provides an independent computation of the bulk viscosity.

Notice that the entropy production rate is quadratic in the bulk scalar field, so the latter can be used in the probe approximation. Neglecting the scalar field backreaction,

$$
\begin{aligned}
& A=-\frac{\dot{a}}{x a}+\frac{1}{4 x^{2} \beta_{2}\left(1-\beta_{2}\right)}\left(1-\sqrt{\left(2 \beta_{2}-1\right)^{2}-\frac{4 x^{4} \beta_{2}\left(\beta_{2}-1\right) \mu^{4}}{a^{4}}}\right), \\
& \Sigma=\frac{a}{x}
\end{aligned}
$$

where we set $x \equiv \frac{1}{r}$. The constant parameter $\mu$ is related to the local temperature $T=$ $T(t)=\frac{\mu}{\pi a(t)}$, and the apparent horizon is located at

$$
r_{h}=\frac{\mu}{a(t)} .
$$

\footnotetext{
${ }^{5}$ We use the same normalization of the couplings on the gravitational side to insure appropriate comparison of the bulk viscosities.
} 
Given (4.7), the equation of motion for the scalar field

$$
\phi=\phi\left(t, z \equiv \frac{\mu x}{a}\right), \quad z \in(0,1),
$$

takes the form

$$
\begin{aligned}
0= & \frac{\partial^{2} \phi}{\partial z^{2}}+\frac{4 a \beta_{2}\left(\beta_{2}-1\right)}{\mu(1-\sqrt{G})} \frac{\partial^{2} \phi}{\partial t \partial z}+\frac{\left(\sqrt{G}(3-\sqrt{G})-2\left(2 \beta_{2}-1\right)^{2}\right)}{z(\sqrt{G}-1) \sqrt{G}} \frac{\partial \phi}{\partial z} \\
& +\frac{6 \beta_{2} a\left(\beta_{2}-1\right)}{z \mu(\sqrt{G}-1)} \frac{\partial \phi}{\partial t}-\frac{2 \Delta(\Delta-4)\left(\beta_{2}-1\right)}{(\sqrt{G}-1) z^{2}} \phi,
\end{aligned}
$$

where

$$
G \equiv\left(2 \beta_{2}-1\right)^{2}-4 z^{4} \beta_{2}\left(\beta_{2}-1\right) .
$$

A general solution of (4.10) can be represented as a series expansion in the successive derivatives of the FLRW boundary metric scalar factor $a(t)$ :

$$
\phi_{\Delta}=\hat{\delta}_{\Delta} a^{4-\Delta} \sum_{n=0}^{\infty} \frac{\mathcal{T}_{\Delta, n}[a]}{\mu^{n}} F_{\Delta, n}(z), \quad \hat{\delta} \equiv \frac{\lambda_{4-\Delta}}{\mu^{4-\Delta}},
$$

with $\mathcal{T}_{\Delta, 0}=1$ and

$$
\mathcal{T}_{\Delta, n}=\frac{1}{4}\left(a \dot{\mathcal{T}}_{\Delta, n-1}+(4-\Delta) \dot{a} \mathcal{T}_{\Delta, n-1}\right), \quad n \geq 1
$$

and

$$
\begin{aligned}
0= & F_{\Delta, 0}^{\prime \prime}+\frac{\sqrt{G}(3-\sqrt{G})-2\left(2 \beta_{2}-1\right)^{2}}{z(\sqrt{G}-1) \sqrt{G}} F_{\Delta, 0}^{\prime}-\frac{2 \Delta(\Delta-4)\left(\beta_{2}-1\right)}{(\sqrt{G}-1) z^{2}} F_{\Delta, 0}, \\
0= & F_{\Delta, n}^{\prime \prime}+\frac{\sqrt{G}(3-\sqrt{G})-2\left(2 \beta_{2}-1\right)^{2}}{z(\sqrt{G}-1) \sqrt{G}} F_{\Delta, n}^{\prime}-\frac{2 \Delta(\Delta-4)\left(\beta_{2}-1\right)}{(\sqrt{G}-1) z^{2}} F_{\Delta, n} \\
& -\frac{16 \beta_{2}\left(\beta_{2}-1\right)}{\sqrt{G}-1}\left(F_{\Delta, n-1}^{\prime}-\frac{3}{2 z} F_{\Delta, n-1}\right), \quad n \geq 1,
\end{aligned}
$$

with boundary conditions

$$
F_{\Delta, 0}=\left\{\begin{array}{ll}
z+\mathcal{O}\left(z^{2}\right), & \Delta=3, \\
z^{2} \ln z^{2}+\mathcal{O}\left(z^{2}\right), & \Delta=2,
\end{array} \quad F_{\Delta, n \geq 1}=\mathcal{O}\left(z F_{\Delta, 0}\right) .\right.
$$

Recursive equations (4.13) can be solved analytically for simple boundary cosmological models [27]. Here, we will be concerned with the de-Sitter expansion at the boundary, i.e., $a(t)=e^{H t}$ ( $H$ being a Hubble constant), in which case

$$
\mathcal{T}_{\Delta, n}=\frac{\Gamma(n+4-\Delta) H^{n} a^{n}}{4^{n} \Gamma(4-\Delta)}, \quad n \geq 0
$$

It is straightforward to verify that the recursive linear ODEs (4.14) reduce in $\beta_{2} \rightarrow 1$ limit to the corresponding equations in [27]. However, for $\beta_{2} \neq 1$ these equations have more 

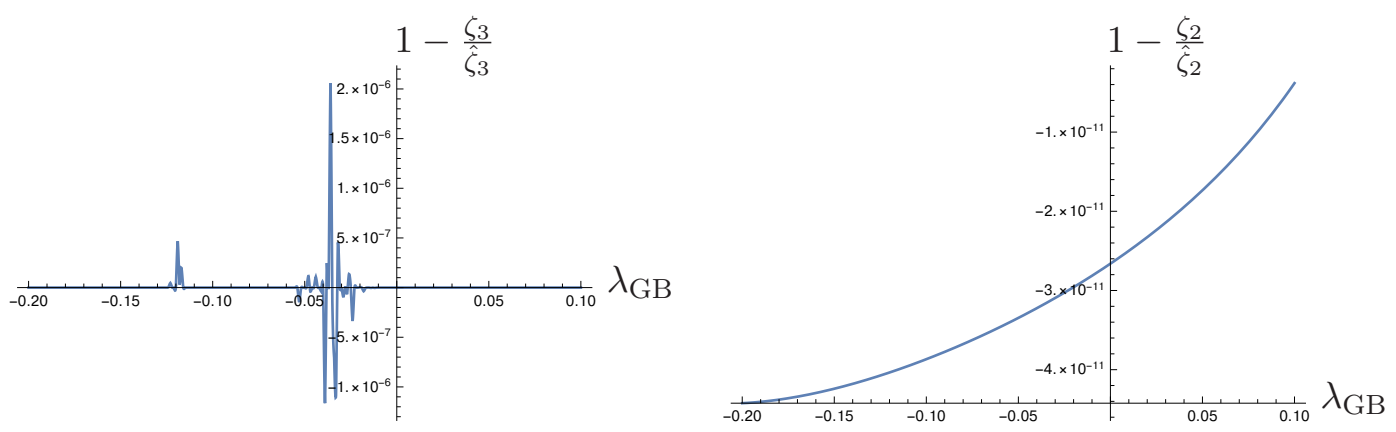

Figure 7. Comparison of the bulk viscosity coefficient $\zeta_{\Delta}$, see (3.7), extracted from the sound waves dispersion relation and the corresponding coefficient $\hat{\zeta}_{\Delta}$, see (4.18), extracted from the leading hydrodynamic contribution in the entropy production rate for the FLRW flow.
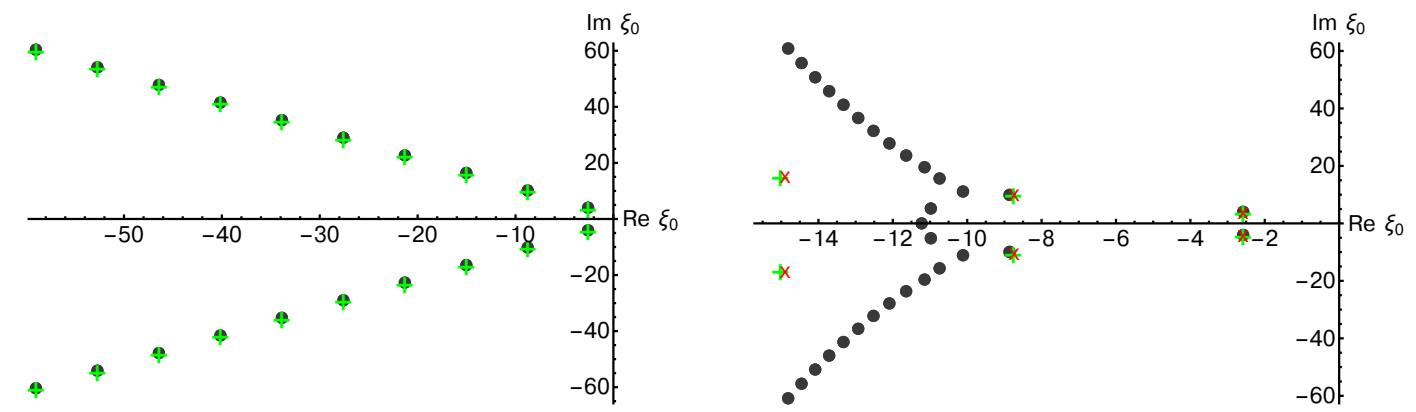

Figure 8. QNMs and leading singularities on the Borel plane for the $\Delta=2$ RG flow with $\beta_{2}=1$ (or $\lambda_{\mathrm{GB}}=0$ ) (left panel) and $\beta_{2}=1.001$ (or $\lambda_{\mathrm{GB}}=-0.001001$ ) (right panel). We used $n_{\max }=300$ terms in the expansion (4.12). See text for the legend.

than three singularities on a Riemann sphere; thus, even for $n=0$ case they can only be solved numerically.

The $n=0$ term in the expansion (4.12) represents the leading hydrodynamic response. Following (4.5), (4.6), (4.7), (4.14) and (4.15) we obtain an elegant expression for the bulk viscosity to the entropy density ratio, to the quadratic order in the coupling constant $\lambda_{\Delta-4}$,

$$
\frac{\zeta}{s}=\frac{\hat{\delta}^{2} a^{8-2 \Delta}(4-\Delta)^{2}\left[F_{\Delta, 0}(z=1)\right]^{2}}{36 \pi}=\frac{(4-\Delta)^{2}\left[F_{\Delta, 0}(z=1)\right]^{2}}{\pi^{9-2 \Delta}}\left(\frac{\lambda_{4-\Delta}}{T^{4-\Delta}}\right)^{2},
$$

correspondingly, using the conformal limit of (3.5),

$$
\frac{\zeta}{\eta}=\left(\frac{\lambda_{4-\Delta}}{T^{4-\Delta}}\right)^{2} \hat{\zeta}_{\Delta}\left(\lambda_{\mathrm{GB}}\right), \quad \hat{\zeta}_{\Delta}=\frac{(4-\Delta)^{2}}{9 \pi^{8-2 \Delta}\left(2 \beta_{2}-1\right)^{2}}\left[F_{\Delta, 0}(z=1)\right]^{2} .
$$

Of course, $\hat{\zeta}_{\Delta}$ should agree precisely with $\zeta_{\Delta}$ in (3.7). Figure 7 demonstrates this agreement. It validates the hydrodynamic computations in section 3 ; it also confirms the conjectured identification of the apparent horizon with the dynamical entropy of the boundary gauge theory in the presence of the bulk GB term.

We conclude this section commenting on the asymptotic properties of the expansion (4.12). As argued in [27], the above expansion is asymptotic at $\beta_{2}=1$, with the poles in the Pade approximates of their Borel transforms with high accuracy reproducing 

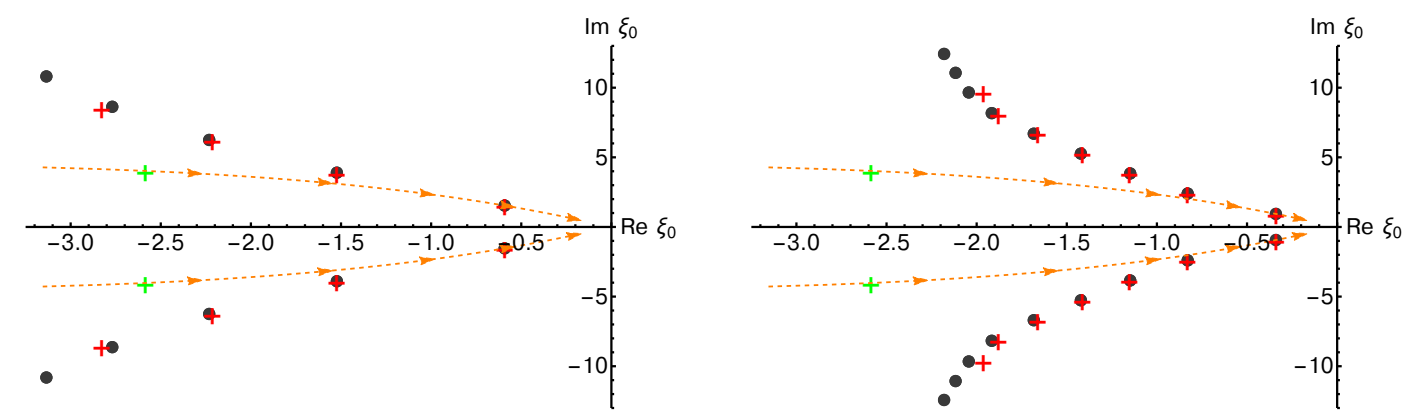

Figure 9. QNMs and leading singularities on the Borel plane for the $\Delta=2 \mathrm{RG}$ flow with $\beta_{2}=3$ (or $\lambda_{\mathrm{GB}}=-6$ ) (left panel) and $\beta_{2}=5$ (or $\lambda_{\mathrm{GB}}=-20$ ) (right panel). We used $n_{\max }=300$ terms in the expansion (4.12). See text for the legend.

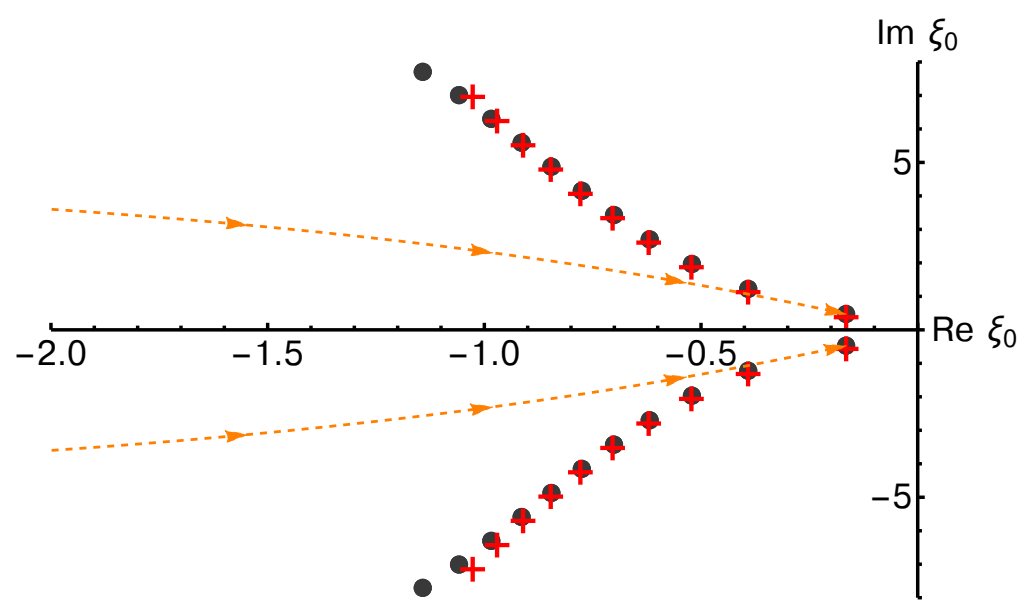

Figure 10. QNMs and leading singularities on the Borel plane for the $\Delta=2 \mathrm{RG}$ flow with $\beta_{2}=10$ (or $\lambda_{\mathrm{GB}}=-90$ ). We used $n_{\max }=300$ terms in the expansion (4.12). See text for the legend.

the corresponding QNM spectra, i.e., the spectra of non-hydrodynamic excitations in the boundary gauge theory plasma. Here, we have an opportunity to study the interplay of the convergence of the expansion (4.12) and the micro-causality of the model (2.4). We focus on $\Delta=2$ RG flows. ${ }^{6}$ The results are summarized in figures 8-10:

- The solid black circles represent the leading singularities $\xi_{0}$ on the complex plane closest to the origin for the Borel transform of the expansion (4.12). ${ }^{7}$ Green crosses correspond to QNM frequencies for $\Delta=2$ and $\beta_{2}=1$ taken from [37] and redefined according to $\omega_{\mathrm{QNM}}(T)=\hat{\omega}_{\mathrm{QNM}} T$ and $\xi_{0}=-i \hat{\omega}_{\mathrm{QNM}}$. Red crosses represent QNM frequencies for $\Delta=2$ and $\beta_{2}=\{1.001,3,5,10\}$ (figures 8 (right panel), 9 (left panel), 9 (right panel), 10) correspondingly. Directed orange dashed curves trace the 'flows' of the lowest QNM mode at $\beta_{2}=1$ to the corresponding value of $\beta_{2}$. In figure 9 the orange flows are extended to $\beta_{2}<1\left(\lambda_{\mathrm{GB}}>0\right)$ to illustrate that our computation of the QNMs agrees with results of [37] at $\beta_{2}=1$.

\footnotetext{
${ }^{6}$ There is no qualitative difference for the case of $\Delta=3$.

${ }^{7}$ Details of the Borel transform, Pade approximation, etc., can be found in [27].
} 
- The left panel of figure 8 reproduces the results of [27] at $\lambda_{\mathrm{GB}}=0$. Computations with small but non-zero $\lambda_{\mathrm{GB}}$ are rather challenging - the direct substitution $\beta_{2}=1$ in (4.14) is singular, and taking the limit (which as is not singular) substantially degrades the numerical accuracy. This problem disappears as $\beta_{2}$ deviates substantially from 1. Results presented in the right panel of figure 8 realize $\lambda_{\mathrm{GB}}=-0.001001$. Because the GB coupling is small, there is almost no flow for the QNMs: the red and green crosses are on top of each other. Here, we recover only the two lowest QNMs. We do not believe that 'unmatched' solid circles represent additional QNMs that disappear in the limit $\beta_{2} \rightarrow 1$ (we did not find extra QNMs).

- figure 9 presents the model with $\beta_{2}=3$ (left panel) and $\beta_{2}=5$ (right panel). Here we reproduce couple more QNMs (red crosses on top of solid circles). Notice that as $\beta_{2}$ increases ( $\lambda_{\mathrm{GB}}$ becomes more negative), the singularities on the Borel plane accumulate. We traced (orange curves) the lowest QNM at $\lambda_{\mathrm{GB}}=0$ (green cross) to the lowest QNM/leading Borel singularity at corresponding $\lambda_{\mathrm{GB}}\left(\lambda_{\mathrm{GB}}=-6\right.$ for the left panel and $\lambda_{\mathrm{GB}}=-20$ for the right panel). We verified that one can trace in a similar fashion higher QNMs as well. Again, we see no indication of additional QNMs that are removed from the spectra as $\beta_{2} \rightarrow 1$.

- figure 10 presents the model with $\beta_{2}=10$. The general trend observed in figure 9 continues: there is even better agreement between the Borel plane singularities and the QNMs; the singularities accumulate.

- We do not present results with $\lambda_{\mathrm{GB}}>0$ : they are qualitatively similar to the case of the small negative GB coupling discussed above. The reason for that is that $0<\lambda_{\mathrm{GB}}<\frac{1}{4}$ (correspondingly $1>\beta_{2}>\frac{1}{2}$, i.e., $\beta_{2} \sim 1$ ) is required for the standard gauge/gravity dictionary, and at $\lambda_{\mathrm{GB}}=\frac{1}{4}$ the GB gravity becomes topological [38].

Note: whether or not the model is micro-causal, its hydrodynamic expansion is always asymptotic.

\section{Causality}

Consider a plasma at thermodynamic equilibrium. There will be a spectrum of fluctuations in the plasma, with the dispersion relation $\mathfrak{w}=\mathfrak{w}(\mathfrak{q})$. The speed with which a wave-front propagates out from a discontinuity in any initial data is governed by [39-41]

$$
\lim _{|\mathfrak{q}| \rightarrow \infty} \frac{\operatorname{Re}(\mathfrak{w})}{\mathfrak{q}}=v^{\text {front }}
$$

The statement of the microscopic causality of the theory is the statement that for all the fluctuations (typically there are multiple branches/channels in the spectrum)

$$
v^{\text {front }} \leq 1
$$

In the framework of gauge/gravity correspondence, the physical fluctuations in the plasma are encoded in the spectrum of the QNMs of the black hole/black brane holographically 
representing the thermal equilibrium state of the latter. For conformal examples of the correspondence with the boundary gauge theory having $c-a \neq 0$ micro-causality analysis where performed in $[28,30]$ leading to the constraint (2.4) in GB gravitational models. Here we would like to extend the results to non-conformal GB models introduced in section 2.

Notice that the question of micro-causality is the question of the deep UV properties of the theory, thus one expects that breaking the scale invariance with a relevant or marginal deformation, i.e., with a dimension $\Delta \leq 4$ operator, should not affect the result (2.4). Causality should not depend on the state of the theory, ${ }^{8}$ for example, the temperature compare to the coupling strength $\lambda_{4-\Delta}$. However, in principle,

- If several relevant couplings are present, causality can be affected by the dimensionless ratio of these couplings. ${ }^{9}$

- Additionally, recall [28] that different channels of the fluctuations in plasma affect causality differently: the scalar channel of the bulk graviton fluctuations constraints

$$
\lambda_{\mathrm{GB}} \leq \lambda_{\mathrm{GB}}^{\mathrm{scalar}}=\frac{9}{100},
$$

while the shear and the sound channels constraint correspondingly:

$$
\lambda_{\mathrm{GB}} \geq \lambda_{\mathrm{GB}}^{\text {shear }}=-\frac{3}{4}, \quad \lambda_{\mathrm{GB}} \geq \lambda_{\mathrm{GB}}^{\text {sound }}=-\frac{7}{36} .
$$

It is only the union of all the constraints that determines (2.4). If the theory is nonconformal, obviously, there is a spectrum of operators present at its UV fixed point, which coupling constants can be adjusted. Existence of these operators introduces additional fluctuation channels (additional branches of the QNMs) which can further constraint the microscopic causality of the model.

In the section we investigate the second of the possibilities mentioned above. To this end, consider the branch of the QNMs of the 'conformal' black brane geometry, i.e., (2.5) with (2.9) for the metric warp factors, associated with the fluctuations of the bulk scalar field, dual to a dimension $\Delta \leq 4$ operator. Following [28], this quasinormal mode equation can be rewritten in the form of the Schrödinger equation:

$$
\begin{aligned}
-\hbar^{2} \partial_{y}^{2} \psi_{[\Delta]}+U_{[\Delta]} \psi_{[\Delta]} & =\alpha^{2} \psi_{[\Delta]}, \quad \hbar \equiv \frac{1}{\mathfrak{q}}, \quad \alpha=\frac{\mathfrak{w}}{\mathfrak{q}}, \\
\text { where } U_{[\Delta]} & =U_{[\Delta]}^{0}+\hbar^{2} U_{[\Delta]}^{1} .
\end{aligned}
$$

The first part of the effective potential has the simple form when expressed in terms of $x$,

$$
U_{[\Delta]}^{0}(x)=\frac{\sqrt{\left(2 \beta_{2}-1\right)^{2}-4 \beta_{2}\left(\beta_{2}-1\right) x^{2}}-1}{2\left(\beta_{2}-1\right)} .
$$

while the expression for $U_{[\Delta]}^{1}$ is too long to be presented here, but we note that the latter is a function only of $x, \beta_{2}, \Delta$ and $\alpha$. What is important is that in the limit $\mathfrak{q} \rightarrow \infty$ (or

\footnotetext{
${ }^{8}$ We explicitly verified this statement in our models.

${ }^{9}$ We can not probe this in our GB models, as we have a single relevant deformation which is treated in the probe approximation.
} 
$\hbar \rightarrow 0$ ), everywhere except in the tiny region $y \gtrsim-\frac{1}{\mathfrak{q}}$ the dominant contribution to $U_{[\Delta]}$ comes from $U_{[\Delta]}^{0}$. Thus in this limit we simply replace

$$
\hbar^{2} U_{[\Delta]}^{1}= \begin{cases}0 & y<0 \\ +\infty & y \geq 0\end{cases}
$$

Causality is violated if the effective Schrödinger problem has a bound state with $\alpha^{2}>1$. It is easy to see that such a bound state does not exist for any value of $\beta_{2}$ since $U_{[\Delta]}^{0}$ is a monotonically decreasing function of $x$ from 1 to 0 . We conclude that the spectrum of operators of a GB CFT (besides the stress-energy tensor) does not further constraint its causal properties beyond (2.4).

\section{Conclusion}

In this work we summarized some (near-)equilibrium properties of the (phenomenological) holographic RG flows with a dual four-dimensional gauge theory interpretation. The UV fixed point of the theory has different central charges, i.e., $c-a \neq 0$, and the flow is triggered by the relevant operator $\mathcal{O}_{\Delta}$ with dimension $\Delta=\{2,3\}$. We considered RG flows close to the UV fixed, i.e., the mass scale associated with the coupling constant of the conformal symmetry breaking deformation, $\lambda_{4-\Delta}$, is much smaller that the local temperature of the boundary gauge theory plasma, see (1.2). We worked to leading nontrivial order in the (explicit) conformal symmetry breaking parameter, but for arbitrary finite values of $c-a$. The simple gravitational model capturing the physics is that of the five-dimensional GB gravity with a minimally coupled bulk scalar field of the appropriate mass, see (2.1).

To summarize:

- We presented holographic renormalization of the model, sufficient to compute the one- and two-point thermal correlation functions of the stress-energy tensor and $\mathcal{O}_{\Delta}$.

- We computed equation of the state, the transport properties (the speed of sound waves, the shear and bulk viscosities), and studied the large-order hydrodynamic gradient expansion in our GB plasma. We discussed the micro-causality of the model.

- Particular attention was devoted towards consistencies of the computations: the holographic renormalization was checked testing the first law of thermodynamics (see figure 2); the speed of sound waves was computed from the equation of state (2.18) and compared with leading-order term in the sound-channel QNMs dispersion relation (3.6) (see figure 4); the bulk viscosity was extracting from the sound waves dispersion relation (3.6) and compared with the bulk viscosity obtained from the entropy growth rate for the homogeneous and isotropic expansion of the plasma (4.6) (see figure 7) - notice that because the attenuation of sound waves depends on the shear viscosity of the plasma as well, we are indirectly testing here the consistency of the shear viscosity computations from the sound waves and from the viscosity Kubo formula (3.4). We verified that our transport coefficients (and the spectrum of 
the non-hydrodynamic modes in plasma) at $c=a$ agrees with the results (whenever available) in the literature.

- We presented a simple and compact formula, see (4.18), for the bulk viscosity from the entropy growth rate in GB model, reminiscent to the Eling-Oz formula $[42,43]$.

- We argued that non-conformal deformations of a holographic CFT with $c-a \neq 0$ do not effect the causal properties of the theory - allowed range of the GB coupling constant (or the difference of the central charged at the UV fixed point) is still given by (2.4).

- We showed that the bulk viscosity bound introduced in [33] is violated for sufficiently large $a-c>0$. This should be contrasted with the shear viscosity bound [35] which is violated for arbitrary small $c-a>0$.

- Conformal field theories have vanishing $\delta_{c_{s}} \equiv \frac{1}{3}-c_{s}^{2}$ and $\delta_{\zeta} \equiv \frac{\zeta}{\eta}$. In non-conformal RG flows both $\delta_{c_{s}}$ and $\delta_{\zeta}$ do not vanish: while the former has a rather mild dependence on $\lambda_{\mathrm{GB}}$ in the causal windows ( $\delta_{c_{s}}$ varies by $\sim 15 \%$ for $\Delta=2$ deformation, and by $\sim 8 \%$ for $\Delta=3$ deformation ), the variation of the latter is more substantial ( $\delta_{\zeta}$ varies by $\sim 50 \%$ for $\Delta=2$ deformation, and by $\sim 60 \%$ for $\Delta=3$ deformation). Shear viscosity does not vanish in the conformal limit and varies by $\sim 80 \%$ in the causal window.

- We showed that the hydrodynamic expansion in non-conformal GB models is an asymptotic series, whether or no the model is microscopically causal. As in [27], for $\lambda_{\mathrm{GB}} \neq 0$ (or $c-a \neq 0$ ) the leading singularities on the Borel plane for non-conformal RG flows agree with the corresponding QNMs - the agreement improves as $\left(-\lambda_{\mathrm{GB}}\right)$ becomes larger. We observe accumulation of the singularities close to the origin for large $a-c>0$. Our analysis support the physical picture advocated in the original work [44] that the asymptotic properties of the hydrodynamic gradient expansion are controlled by the non-hydrodynamic modes, with the lowest lying modes being the most important. Thus our results are not surprising: low-lying states in the spectrum of non-hydrodynamic excitations in plasma do not probe the micro-causality of the model. This picture has further nice confirmation in the recent work [45]. Here, the full hydrodynamic gradient expansion truncates at the second order (being obviously a convergent series) because the limit of the large number of spatial dimensions decouples (removes from the spectrum) the non-hydrodynamics plasma excitations (non-hydrodynamic QNMs in the dual gravitational description).

\section{Acknowledgments}

Research at Perimeter Institute is supported by the Government of Canada through Industry Canada and by the Province of Ontario through the Ministry of Research \& Innovation. This work was further supported by NSERC through the Discovery Grants program. 
Open Access. This article is distributed under the terms of the Creative Commons Attribution License (CC-BY 4.0), which permits any use, distribution and reproduction in any medium, provided the original author(s) and source are credited.

\section{References}

[1] J.M. Maldacena, The large-N limit of superconformal field theories and supergravity, Int. J. Theor. Phys. 38 (1999) 1113 [Adv. Theor. Math. Phys. 2 (1998) 231] [hep-th/9711200] [INSPIRE].

[2] O. Aharony, S.S. Gubser, J.M. Maldacena, H. Ooguri and Y. Oz, Large-N field theories, string theory and gravity, Phys. Rept. 323 (2000) 183 [hep-th/9905111] [InSPIRE].

[3] D.T. Son and A.O. Starinets, Viscosity, black holes, and quantum field theory, Ann. Rev. Nucl. Part. Sci. 57 (2007) 95 [arXiv:0704.0240].

[4] P.M. Chesler and L.G. Yaffe, Numerical solution of gravitational dynamics in asymptotically Anti-de Sitter spacetimes, JHEP 07 (2014) 086 [arXiv:1309.1439] [INSPIRE].

[5] A. Buchel, J.T. Liu and A.O. Starinets, Coupling constant dependence of the shear viscosity in $N=4$ supersymmetric Yang-Mills theory, Nucl. Phys. B 707 (2005) 56 [hep-th/0406264] [INSPIRE].

[6] P. Benincasa and A. Buchel, Transport properties of $N=4$ supersymmetric Yang-Mills theory at finite coupling, JHEP 01 (2006) 103 [hep-th/0510041] [INSPIRE].

[7] A. Buchel, Resolving disagreement for $\eta / s$ in a CFT plasma at finite coupling, Nucl. Phys. B 803 (2008) 166 [arXiv:0805. 2683] [INSPIRE].

[8] A. Buchel and M. Paulos, Relaxation time of a CFT plasma at finite coupling, Nucl. Phys. B 805 (2008) 59 [arXiv:0806.0788] [INSPIRE].

[9] A. Buchel and M. Paulos, Second order hydrodynamics of a CFT plasma from boost invariant expansion, Nucl. Phys. B 810 (2009) 40 [arXiv:0808.1601] [INSPIRE].

[10] Y. Kats and P. Petrov, Effect of curvature squared corrections in AdS on the viscosity of the dual gauge theory, JHEP 01 (2009) 044 [arXiv:0712.0743] [INSPIRE].

[11] A. Buchel, R.C. Myers and A. Sinha, Beyond $\eta / s=1 / 4 \pi$, JHEP 03 (2009) 084 [arXiv: 0812.2521] [INSPIRE].

[12] S. Waeber, A. Schäfer, A. Vuorinen and L.G. Yaffe, Finite coupling corrections to holographic predictions for hot QCD, JHEP 11 (2015) 087 [arXiv:1509.02983] [INSPIRE].

[13] S. Grozdanov, N. Kaplis and A.O. Starinets, From strong to weak coupling in holographic models of thermalization, JHEP 07 (2016) 151 [arXiv:1605.02173] [INSPIRE].

[14] S. Grozdanov and A.O. Starinets, Second-order transport, quasinormal modes and zero-viscosity limit in the Gauss-Bonnet holographic fluid, JHEP 03 (2017) 166 [arXiv: 1611.07053] [INSPIRE].

[15] T. Andrade, J. Casalderrey-Solana and A. Ficnar, Holographic isotropisation in Gauss-Bonnet gravity, JHEP 02 (2017) 016 [arXiv: 1610.08987] [INSPIRE].

[16] J. Casalderrey-Solana, N.I. Gushterov and B. Meiring, Resurgence and hydrodynamic attractors in Gauss-Bonnet holography, arXiv:1712.02772 [INSPIRE]. 
[17] D.J. Gross and E. Witten, Superstring modifications of Einstein's equations, Nucl. Phys. B 277 (1986) 1 [INSPIRE].

[18] M.B. Green and C. Stahn, D3-branes on the Coulomb branch and instantons, JHEP 09 (2003) 052 [hep-th/0308061] [INSPIRE].

[19] M.F. Paulos, Higher derivative terms including the Ramond-Ramond five-form, JHEP 10 (2008) 047 [arXiv: 0804.0763] [INSPIRE].

[20] S. Nojiri and S.D. Odintsov, On the conformal anomaly from higher derivative gravity in AdS/CFT correspondence, Int. J. Mod. Phys. A 15 (2000) 413 [hep-th/9903033] [inSPIRE].

[21] M. Blau, K.S. Narain and E. Gava, On subleading contributions to the AdS/CFT trace anomaly, JHEP 09 (1999) 018 [hep-th/9904179] [INSPIRE].

[22] X.O. Camanho, J.D. Edelstein, J. Maldacena and A. Zhiboedov, Causality constraints on corrections to the graviton three-point coupling, JHEP 02 (2016) 020 [arXiv:1407.5597] [INSPIRE].

[23] A. Buchel, L. Lehner and R.C. Myers, Thermal quenches in $N=2^{*}$ plasmas, JHEP 08 (2012) 049 [arXiv: 1206.6785] [INSPIRE].

[24] R. Baier, P. Romatschke, D.T. Son, A.O. Starinets and M.A. Stephanov, Relativistic viscous hydrodynamics, conformal invariance and holography, JHEP 04 (2008) 100 [arXiv:0712.2451] [INSPIRE].

[25] P. Benincasa, A. Buchel and A.O. Starinets, Sound waves in strongly coupled non-conformal gauge theory plasma, Nucl. Phys. B 733 (2006) 160 [hep-th/0507026] [INSPIRE].

[26] A. Buchel and C. Pagnutti, Bulk viscosity of $N=2^{*}$ plasma, Nucl. Phys. B $\mathbf{8 1 6}$ (2009) 62 [arXiv: 0812.3623] [INSPIRE].

[27] A. Buchel, M.P. Heller and J. Noronha, Entropy production, hydrodynamics and resurgence in the primordial quark-gluon plasma from holography, Phys. Rev. D 94 (2016) 106011 [arXiv: 1603.05344] [INSPIRE].

[28] A. Buchel and R.C. Myers, Causality of holographic hydrodynamics, JHEP 08 (2009) 016 [arXiv:0906.2922] [INSPIRE].

[29] R.C. Myers, M.F. Paulos and A. Sinha, Holographic studies of quasi-topological gravity, JHEP 08 (2010) 035 [arXiv: 1004.2055] [INSPIRE].

[30] D.M. Hofman and J. Maldacena, Conformal collider physics: energy and charge correlations, JHEP 05 (2008) 012 [arXiv: 0803.1467] [INSPIRE].

[31] A. Buchel and S. Cremonini, Viscosity bound and causality in superfluid plasma, JHEP 10 (2010) 026 [arXiv: 1007.2963] [InSPIRE].

[32] A. Buchel and J.T. Liu, Universality of the shear viscosity in supergravity, Phys. Rev. Lett. 93 (2004) 090602 [hep-th/0311175] [INSPIRE].

[33] A. Buchel, Bulk viscosity of gauge theory plasma at strong coupling, Phys. Lett. B 663 (2008) 286 [arXiv:0708.3459] [InSPIRE].

[34] A. Buchel, Violation of the holographic bulk viscosity bound, Phys. Rev. D 85 (2012) 066004 [arXiv: 1110.0063] [INSPIRE].

[35] P. Kovtun, D.T. Son and A.O. Starinets, Viscosity in strongly interacting quantum field theories from black hole physics, Phys. Rev. Lett. 94 (2005) 111601 [hep-th/0405231] [INSPIRE]. 
[36] A. Buchel and A. Karapetyan, De Sitter vacua of strongly interacting QFT, JHEP 03 (2017) 114 [arXiv: 1702.01320] [INSPIRE].

[37] A. Núñez and A.O. Starinets, AdS/CFT correspondence, quasinormal modes and thermal correlators in $N=4$ SYM, Phys. Rev. D 67 (2003) 124013 [hep-th/0302026] [INSPIRE].

[38] A.H. Chamseddine, Topological gauge theory of gravity in five-dimensions and all odd dimensions, Phys. Lett. B 233 (1989) 291 [INSPIRE].

[39] L. Brillouin, Wave Propagation and Group Velocity, Academic Press, New York U.S.A. (1960).

[40] R. Fox, C.G. Kuper and S.G. Lipson, Faster-than-light group velocities and causality violation, Proc. Roy. Soc. Lond. A 316 (1970) 515.

[41] E. Krotscheck and W. Kundt, Causality criteria, Commun. Math. Phys. 60 (1978) 171.

[42] C. Eling and Y. Oz, A novel formula for bulk viscosity from the null horizon focusing equation, JHEP 06 (2011) 007 [arXiv:1103.1657] [INSPIRE].

[43] A. Buchel, On Eling-Oz formula for the holographic bulk viscosity, JHEP 05 (2011) 065 [arXiv: 1103.3733] [INSPIRE].

[44] M.P. Heller, R.A. Janik and P. Witaszczyk, Hydrodynamic gradient expansion in gauge theory plasmas, Phys. Rev. Lett. 110 (2013) 211602 [arXiv:1302.0697] [INSPIRE].

[45] M. Rozali, E. Sabag and A. Yarom, Holographic turbulence in a large number of dimensions, arXiv: 1707.08973 [INSPIRE]. 